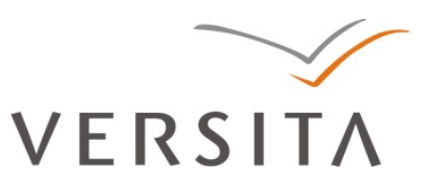

BULGARIAN ACADEMY OF SCIENCES

CYBERNETICS AND INFORMATION TECHNOLOGIES • Volume 13, No 1

Sofia • 2013

Print ISSN: 1311-9702; Online ISSN: 1314-4081

DOI: 10.2478/cait-2013-0004

\title{
Codes Correcting Limited Patterns of Random Errors Using S-K Metric
}

\author{
Bhu Dev Sharma, Ankita Gaur \\ Department of Mathematics, JIIT, Noida, UP-201307, India \\ Emails:bhudev_sharma@yahoo.com_bhudev.sharma@jiit.ac.in ankitagaur50@yahoo.com
}

Abstract: Coding is essential in all communications and in all multi-operation devices, and errors do occur. For error control, the method in vogue is to use code words with redundant digits. The number of redundant digits is determined based on two things - the number of messages and the kind of errors that need to be controlled. For efficient coding the redundant digits have to be kept to the minimum.

In this paper we introduce the idea of limited error patters while using the code alphabet $Z_{q}=\{0,1,2, \ldots, q-1\}$, $\bmod q$, when $q>3$. We define limitations of the errors in a position by substitution of the character there by a specified number of other characters, rather than by any other character. This is not possible through Hamming approach, because there a character in an error could be substituted by any other of the q-1 characters. The firm mathematical base is provided by use of a metric from the class of S-K metrics, Hamming metric being one of these.

The paper gives upper bounds on the codeword lengths for various kinds of "random limited error patterns". Examples and discussion bring out the tremendous improvement and generalization of Rao Hamming bound.

Keywords: Hamming distance, S-K metric, error patterns, error control in codes, bounds. 


\section{Introduction}

With the advancement of information technology, coding theory is having new challenges. This is because the communication channels, the automata or the electronic devices, where they find use have varying characteristics. The errors patterns differ. Theory of error control coding started simply with binary code words of fixed length, with basically three parameters, namely the number information digits, the check digit and the Hamming distance between $n$-tuples. The errors considered were the random errors and the burst errors. When studies were extended to q-nary case, these things continued without further necessary refinement. A single error continued to be an error in any position of any magnitude. The cause for this can be traced in the inbuilt nature of Hamming distance. Lee distance [1], yet another distance is also fixed in nature, with limited scope for study. The work progressed mainly in two directions, these related to optimality considerations and construction of codes capable of correcting a certain number of random error correcting codes. Practically all designed distance codes, $\mathrm{BCH}$, Goppa, Helgert, turned out to be "bad", with rate falling with increasing length, that is asymptotically [1].

Since error control coding now is not limited to distant communication only, mathematics was needed which can suitably match the characteristics of the device for which the coding was required and can consider the correction of only those patterns that need to be corrected rather than the wasted capacity of the correcting non-errors by default.

$\mathrm{S} \mathrm{h}$ a r $\mathrm{m}$ a and $\mathrm{K}$ a u s h i $\mathrm{k}[4,5]$ investigated the question of other possible different ways of defining distances/metrics (S-K distances/metrics) over $Z_{q}=\{0,1,2, \ldots, q-1\}, \bmod q$, when $q>2$. They found a whole class of possible distances defined over $Z_{q}$, by considering partitions of $Z_{q}$, satisfying certain conditions that we take up in the next section.

This class of S-K distances, beside being rich in choice for properly matching the channel, provides possibilities of handling a wide variety of errors, which was not possible with Hamming distance or even with Lee distance considerations. Hamming and Lee metrics are in fact the particular rather extremal cases of S-K studies, refer to [4].

With nature of the errors vastly different and S-K metric, it is possible to consider codes which will have well defined error characteristics and will be far more efficient than otherwise possible with Hamming distance consideration. Since only those errors patterns of a given weight, that form a part of the total error patterns of that weight, are considered for correction, we call them "codes correcting partial errors”. This is undertaken in this paper.

The paper is organized as follows: Section 2 is preliminary, where we recapitulate basic concepts, definitions and results required for later studies. In Section 3 we introduce the idea of partial error correction and obtain lower bounds for a variety of partial error correction. Section 4 gives examples of the partial error correcting codes. 


\section{Preliminaries}

We shall be considering vectors over $Z_{q}=\{0,1,2, \ldots, q-1\}$, the ring of integer, $\bmod q$, where in general in our study, $q>3$.

Definition 1. Hamming Weight and Distance: Given a vector $u=\left(a_{1}, a_{2}, \ldots, a_{n}\right)$ over $Z_{q}$, the Hamming weight of vector $u$ is the number of its nonzero components.

Next, given two $n$-vectors $u=\left(a_{1}, a_{2}, \ldots, a_{n}\right)$ and $v=\left(b_{1}, b_{2}, \ldots, b_{n}\right)$ the Hamming distance between $u$ and $v$ is the number of components, in which the two $n$-tuples differ or is the weight of their component-wise difference vector.

Definition 2. Sharma-Kaushik (S-K) Partition: Let us consider a partition of $Z_{q}$, into (disjoint, nonempty) subsets $B_{0}, B_{1}, \ldots, B_{m-1}$, where $m$ is an integer greater than or equal to 2 , such that

$$
\begin{gathered}
B_{0}=\{0\} ; \\
\text { for } i \in Z_{q}, i \in B_{s} \Leftrightarrow q-i \in B_{s} ;
\end{gathered}
$$

(iii) if $i \in B_{s}$, and $j \in B_{t}$, and $s>t$ (in the order of their natural occurrence in $\left.Z_{q}\right)$

then

$$
\min \{i, q-i\}>\min \{j, q-j\} ;
$$

(iv) if $s>. t,\left|B_{s}\right| \geq\left|B_{t}\right|$, except for $s=m-1$, in which case we may have

$$
\left|B_{m-1}\right| \geq \frac{1}{2}\left|B_{m-2}\right|,
$$

where $|B|=$ number of elements in the set $B$.

Note: When $m=2$, we will call it Hamming partition of $Z_{q}$. Also when each $\left|B_{s}\right|=2$, for $0<s<m-2$, the partition will be called Lee partition, since in that case it leads to Lee-metric, refer to B e r l e k e m p [1].

\section{Definition 3. S-K weights and distances:}

$\boldsymbol{S}-\boldsymbol{K}$ weights: It can be mentioned that in S-K scheme of things, the weights and distances are defined in reference to a S-K partition. Thus for different S-K partitions of $Z_{q}$, these, in general, will have different values for the same element of $Z_{q}$, or for a vector over $Z_{q}$.

We first define the S-K weight of an element $j \in Z_{q}$ corresponding to a S-K partition $P=\left\{B_{0}, B_{1}, \ldots, B_{m-1}\right\}$. Denoting it by $W_{P}(j)$, it is given by

$$
W_{P}(j)=s \text { if } j \in B_{s}, 0 \leq s \leq m-1 .
$$


Next, we define S-K weight $W_{P}(u)$ of $u=\left(a_{1}, a_{2} \ldots, a_{n}\right), \quad a_{i} \in Z_{q}$, corresponding to $\mathrm{S}-\mathrm{K}$ partition $P=\left\{B_{0}, B_{1}, \ldots, B_{m-1}\right\}$, as the sum of the class-weights of its components, i.e.,

$$
W_{P}(u)=\sum_{i=1}^{n} W_{p}\left(a_{i}\right) \text {. }
$$

$S$-K Distance between two vectors: Given two $n$-vectors $u=\left(a_{1}, a_{2}, \ldots, a_{n}\right)$ and $v=\left(b_{1}, b_{2}, . ., b_{n}\right)$, where $a_{i}, b_{i} \in Z_{q}$, the S-K distance between vectors $u$ and $v$ associated with S-K partition $P$ is defined as the sum of the S-K distances between their components, i.e.,

$$
\begin{gathered}
d_{P}(u, v)=\sum_{i=1}^{n} d_{p}\left(a_{i}, b_{i}\right)=\sum_{i=1}^{n} W_{P}\left(a_{i}-b_{i}\right), \\
d_{P}(u, v)=W_{P}(u-v)=\sum_{i=1}^{n} d_{p}\left(a_{i}-b_{i}\right), \text { refer to [2]. }
\end{gathered}
$$

\section{Partial error patterns}

Definition 4. Partial sets: It may be noted that in defining the S-K partition $P=\left\{B_{0}, B_{1}, \ldots, B_{m-1}\right\}$, of $Z_{q}$ arranged in a circular order, $B_{s}$ is really the collection of all elements of $Z_{q}$, on either side of 0 , assigned a distance $s$ from 0 . We can thus call $B_{s}$ a subset or a "partial set”' of $Z_{q}$ at distance $s$ from 0 .

More generally, for an arbitrary element $j \in Z_{q}$ its "partial set at a distance $s$ " is given by $B_{s}(j)=\left\{B_{s}+j\right\}_{q}$, the addition being in each element of $B_{s} \bmod q$.

Example 1. Suppose that we have $Z_{q}=\{0,1,2,3,4,5,6\}$, with $q=7$, and let us consider a $S$-K partition of $Z_{7}$ given that

$$
P=\left\{B_{0}=\{0\}, B_{1}=\{1,6\}, B_{2}=\{2,3,4,5\}\right\} .
$$

We see a partial set of weight 1 for 0 is $B_{1}$ and the "partial set of weight 2 " for 0 is $B_{2}$.

Further the weight-1 partial set of another element, say of 5 , is set of those elements of $Z_{7}$, given by $B_{1}(5)=B_{1}+5=\{1+5,6+5\}_{7}=\{6,4\}$, and $B_{2}(5)=B_{2}+5=\{2+5,3+5,4+5,5+5\}_{7}=\{0,1,2,3\}$.

Note: In this example it may be seen that the other two possible S-K partitions of $Z_{7}$ are

$$
P_{\mathrm{L}}=\left\{B_{0}=\{0\}, B_{1}=\{1,6\}, B_{2}=\{2,5\}, B_{3}=\{3,4\}\right\}
$$


and $P_{\mathrm{H}}=\left\{B_{0}=\{0\}, B_{1}=\{1,2,3,4,5,6\}\right\}$, respectively giving Lee and Hamming distances on $Z_{7}$, in terms of the definitions above. The partial sets of different weights $\left(0,1,2\right.$ and 3 in the case of $P_{\mathrm{L}}$ and of weight 0,1 in the case of $\left.P_{\mathrm{H}}\right)$, the elements in $P_{\mathrm{H}}$ and $P_{\mathrm{L}}$, as can be seen are differently defined.

Definition 5. Patterns of limited errors. It may be easily realized that errors follow a pattern, which differs from a system to system. A system in which an entry in a code word is received as another symbol, the error may be called "substitution error". With S-K scheme of things, it is possible to consider various different limited kinds of substitution errors that were not possible under Hamming scheme of things. Some of the possible ones are illustratively detailed below:

1. Single the error or e-random errors, in which an entry $j$ is substituted by an element of $B_{1}+j$ or some $e$-positions $j_{1}, j_{2}, \ldots, j_{t}$ are similarly substituted correspondingly by an entry with their sum with $B_{1}+j_{1}, B_{1}+j_{2}, \ldots, B_{1}+j_{e}$. We may call a code correcting $e$ such errors " $e B_{1}$-error correcting code", and it must have a minimum distance of $2 e+1$, caused by $B_{1}$ added substitution positions.

2. Single error or e-random errors, in which an entry $j$ is substituted by an element of $B_{2}+j$ or some $e$-positions are similarly substituted by an entry with their sum with $B_{2}$.

One can easily consider different kinds of substitution errors following modifications depending on the partial set (s) that determines the error patterns.

In obtaining a bound on a necessary number of parity-checks for an e-error correcting code, it is customary to define a volume of a sphere of radius e around every code word and consider their mutual disjointness, etc. In the S-K study that we undertake, this idea can be looked more closely and, given an n-vector $u$, we can find numbers of patterns which have a specified $S-K$ distance from the vector $u$.

In the theorems that follow, we consider lower bounds on the number of parity check digits, $r=n-k$.

Theorem 1. Given a S-K partition $P=\left\{B_{0}, B_{1}, \ldots, B_{m-1}\right\}$ of $Z_{q}, q$ prime, a necessary condition for the existence of an $(n, k)$ code over $Z_{q}$, correcting an error in $e$-positions of each of weight one is given by

$$
\sum_{t=0}^{e}\left(\begin{array}{l}
n \\
t
\end{array}\right)\left|B_{1}\right|^{t} \leq q^{n-k} \text { or } n-k \geq \log _{q}\left[\sum_{t=0}^{e}\left(\begin{array}{l}
n \\
t
\end{array}\right)\left|B_{1}\right|^{t}\right] .
$$

P r o o f: It may be recalled that the elements in $B_{1}$ are of S-K weight 1 and that given an entry $j$, an error of weight 1 is obtained by substituting it by an element of $B_{1}+j$. This can happen in $\left|B_{1}\right|$ ways.

Thus, given a code word, the number of vectors at a distance 1 from it are $\left(\begin{array}{l}n \\ 1\end{array}\right)\left|B_{1}\right|$. 
Similarly, the error patterns at a distance 2 are $\left(\begin{array}{l}n \\ 2\end{array}\right)\left|B_{1}\right|^{2}$, and those at a distance $e$ are $\left(\begin{array}{l}n \\ e\end{array}\right)\left|B_{1}\right|^{e}$.

Thus, the total number of vectors that are at a distance of $e$ or less are from a vector $u$ of length $n$ :

$$
\left(\begin{array}{l}
n \\
e
\end{array}\right)\left|B_{1}\right|^{e}+\left(\begin{array}{c}
n \\
e-1
\end{array}\right)\left|B_{1}\right|^{e-1}+\left(\begin{array}{c}
n \\
e-2
\end{array}\right)\left|B_{1}\right|^{e-2}+\ldots+\left(\begin{array}{l}
n \\
1
\end{array}\right)\left|B_{1}\right|+1 \text { or } \sum_{t=0}^{e}\left(\begin{array}{l}
n \\
t
\end{array}\right)\left|B_{1}\right|^{t} .
$$

In order that the code is able to correct $e$-errors of $\mathrm{S}-\mathrm{K}$ weight 1 each, these vectors around any code word should be disjoint, that is for $(n, k)$ code of $q^{k}$ code words,

$$
\begin{aligned}
& q^{k} \sum_{t=0}^{e}\left(\begin{array}{l}
n \\
t
\end{array}\right)\left|B_{1}\right|^{t} \leq q^{n}, \\
& \text { or } \sum_{t=0}^{e}\left(\begin{array}{l}
n \\
t
\end{array}\right)\left|B_{1}\right|^{t} q^{n-k} .
\end{aligned}
$$

This proves the result.

Note: It may be worthwhile to reflect on the idea of partial sets of certain weights around elements of $Z_{q}$. Let us consider the keyboard of a computer, it has keys for various numbers and other symbols. Imagine punching a number or an alphabet key on it. While word processing, one may erroneously strike a key on one or two positions on either side of the right key, rather than any key on the keyboard. These likely positions will constitute the partial set of errors for the number or the symbol key pressed.

Next we want to consider e-random errors, where the substitution errors are caused by elements at distances of 1 or 2, that is from two segments of partial errors are handled for correction.

First we obtain the result in

Lemma 1. Given a S-K partition $P=\left\{B_{0}, B_{1}, \ldots, B_{m-1}\right\}$ of $Z_{q}, q$ prime, and a vector of length $n$, the number of vectors obtained by substituting in $e$-positions, each position $j$ by elements of $B_{1}+j$ or $B_{2}+j$, is given by

$$
\left(\left|B_{1}\right|+\left|B_{2}\right|\right)^{e} \text {. }
$$

P r o o f: Let us consider that out of $e$ positions selected, $k$ of them have entry $j$ that is substituted by an entry from $B_{1}+j$ and the other $e-k$ by entries from $B_{2}+j$.

Then this can be done in $\left(\begin{array}{l}e \\ k\end{array}\right)\left|B_{1}\right|^{k}\left|B_{2}\right|^{e-k}$ ways.

Thus the total, as $k$ varies from 0 to $e$, gives the required number of vectors as 


$$
\begin{gathered}
\left(\left|B_{2}\right|^{e}+\left(\begin{array}{l}
e \\
1
\end{array}\right)\left|B_{1}\right|\left|B_{2}\right|^{e-1}+\left(\begin{array}{l}
e \\
2
\end{array}\right)\left|B_{1}\right|^{2}\left|B_{2}\right|^{e-2}+\ldots+\left(\begin{array}{l}
e \\
k
\end{array}\right)\left|B_{1}\right|^{k}\left|B_{2}\right|^{e-k}+\ldots .+\left|B_{1}\right|^{e}\right) \\
\text { or }\left(\left|B_{1}\right|+\left|B_{2}\right|\right)^{e} .
\end{gathered}
$$

This proves the result.

Note: It can immediately be seen in place of $B_{1}$ and $B_{2}$, that the result will hold for any two distinct or non-distinct partial sets $B_{s}$ and $B_{t}$, Also it can be generalized from just two to any numbers of partial-sets. We state the result for any $s$ partial-sets below:

Lemma 2. Given a S-K partition $P=\left\{B_{0}, B_{1}, \ldots, B_{m-1}\right\}$ of $Z_{q}$, $q$ prime, and a vector of length $n$, the number of vectors obtained by substituting in $e$-positions, each position $j$ by elements of $k$ partial sets $B_{r_{1}}+j, B_{r_{2}}+j, \ldots, B_{r_{s}}+j$, $s \leq m-1$, is given by

$$
\left(\left|B_{r_{1}}\right|+\left|B_{r_{2}}\right|+\ldots .+\left|B_{r_{s}}\right|\right)^{e} .
$$

Theorem 2. Given a S-K partition $\mathrm{P}=\left\{B_{0}, B_{1}, \ldots, B_{m-1}\right\}$ of $Z_{q}, q$ prime, a necessary condition for the existence of an $(n, k)$ code over $Z_{q}$, correcting the error in $e$ or less positions so that in these positions, the entry $j$ when an error is received as an element of $B_{1}+j$ or $B_{2}+j$ is given by

$$
\sum_{t=0}^{e}\left(\begin{array}{l}
n \\
e
\end{array}\right)\left(\left|B_{1}\right|+\left|B_{2}\right|\right)^{t} \leq q^{n-k} \text {. }
$$

P r o of: Now e positions of out $n$ can be chosen in $\left(\begin{array}{l}n \\ e\end{array}\right)$ ways and by the Lemma 2 given, these can undergo defined changes in $\left(\left|B_{1}\right|+\left|B_{2}\right|\right)^{e}$ ways.

Thus, the number of ways any exactly e defined errors can occur, is

$$
\left(\begin{array}{l}
n \\
e
\end{array}\right)\left(\left|B_{1}\right|+\left|B_{2}\right|\right)^{e} .
$$

The total number of the error patterns in $e$ or less positions is then given by

$$
\sum_{t=0}^{e}\left(\begin{array}{l}
n \\
t
\end{array}\right)\left(\left|B_{1}\right|+\left|B_{2}\right|\right)^{t} \text {. }
$$

With this number of error patterns around each code, the word to be disjoint, we get the necessary condition as

$$
\sum_{t=0}^{e}\left(\begin{array}{l}
n \\
t
\end{array}\right)\left(\left|B_{1}\right|+\left|B_{2}\right|\right)^{t} \leq q^{n-k}
$$


Note: Hamming bound case: In the Hamming case, the partition of $Z_{q}$ is two partial sets $B_{0}=\{0\}$, and $B_{1}=\{0,1, \ldots, q-1\}$, so that $\left|B_{1}\right|=q-1$, and the result of these theorems reduces to the famous Rao Hamming bound [3].

Example 2. Let $Z_{q}=\{0,1,2,3,4,5,6,7,8,9,10,11,12\}, q=13$; to illustrate the results of the above two theorems we consider two S-K partitions of $Z_{q}$.

Case $\mathrm{A}$. Let us first consider the $\mathrm{S}-\mathrm{K}$ partition given by

$$
P_{1}=\left\{B_{0}, B_{1}, B_{2}, B_{3}\right\} \text {, }
$$

where

$$
B_{0}=\{0\}, B_{1}=\{1,12\}, B_{2}=\{2,3,4,9,10,11\} \text {, and } B_{3}=\{5,6,7,8\} .
$$

For an $(n, k)$ code correcting single error of weight 1 , from Theorem 1 , we have the bound

$$
\begin{gathered}
1+\left(\begin{array}{l}
n \\
1
\end{array}\right)\left|B_{1}\right| \leq q^{n-k} \text {, where } n-k=r \\
\text { or } n \leq \frac{q^{r}-1}{\left|B_{1}\right|} .
\end{gathered}
$$

For different values of $r$, here $\left|B_{1}\right|=2$, the upper-bound on $n$ for $(n, k)$, $k=n-r$, is given in Table 1 . In the table, we have compared the limited $\left|B_{1}\right|$ single error correction bound by a Hamming single error correcting bound. The gains are quite astonishing, for example for $r=3$, these results tell us that the numbers of messages for $\left|B_{1}\right|$ error correction can use $13^{1095}$ messages while under -Hamming criterion, this number is a small fraction $13^{180}$, and this proportion increases exponentially.

For calculation of the rate, we have used the usual ratio, $R=\frac{k}{n}$.

Table 1

\begin{tabular}{|l|l|l|l|l|l|l|}
\hline \multirow{2}{*}{$r$} & \multicolumn{3}{|c|}{$\begin{array}{c}\text { Upper bound on } n \\
\text { with a S-K single error }\end{array}$} & \multicolumn{3}{c|}{$\begin{array}{c}\text { Upper bound on } n \\
\text { with a Hamming-single error }\end{array}$} \\
\cline { 2 - 7 } & $n$ & $k$ & $R$ & $N$ & $k$ & $R$ \\
\hline 1 & 6 & 5 & 0.83333 & 1 & 0 & 0 \\
\hline 2 & 84 & 82 & 0.97619 & 14 & 12 & 0.85714 \\
\hline 3 & 1098 & 1095 & 0.99727 & 183 & 180 & 0.983606 \\
\hline 4 & 14280 & 14276 & 0.99972 & 2830 & 2826 & 0.998586 \\
\hline 5 & 185646 & 185641 & 0.99997 & 30941 & 30936 & 0.9998384 \\
\hline 6 & 2413404 & 2413398 & 0.999998 & 402234 & 402228 & 0.9999850 \\
\hline 7 & 31374258 & 31374251 & 0.9999998 & 522943 & 5229036 & 0.999998661 \\
\hline
\end{tabular}

Case B. Next we consider, for the S-K partition $P_{1}$, bounds on $n$ for code correcting errors in a single position which are either of S-K weight 1 or S-K weight 2. From Theorem 2 this bound is given by 


$$
1+\left(\begin{array}{l}
n \\
1
\end{array}\right)\left(\left|B_{1}\right|+\left|B_{2}\right|\right) \leq q^{n-k} \text { or } n \leq \frac{q^{r}-1}{\left(\left|B_{1}\right|+\left|B_{2}\right|\right)} .
$$

In the example when $q=13$, for different values of $r$ here $\left(\left|B_{1}\right|+\left|B_{2}\right|\right)=8$, the upper-bound on $n$ for $(n, k), r=n-k$, is given in Table 2 .

Table 2

\begin{tabular}{|l|l|l|l|l|l|l|}
\hline \multirow{2}{*}{$r$} & \multicolumn{3}{|c|}{$\begin{array}{c}\text { Upper bound on } n \\
\text { with S-K single error }\end{array}$} & \multicolumn{3}{c|}{$\begin{array}{c}\text { Upper bound on } n \\
\text { with }\end{array}$} \\
\cline { 2 - 7 } & $n$ & $k$ & $R$ & $n$ & $k$ & $R$ \\
\hline 1 & 1 & 0 & 0 & 1 & 0 & 0 \\
\hline 2 & 21 & 19 & 0.90476 & 14 & 12 & 0.85714 \\
\hline 3 & 274 & 271 & 0.989051094 & 183 & 180 & 0.983606 \\
\hline 4 & 3570 & 3566 & 0.998879551 & 2830 & 2826 & 0.998586 \\
\hline 5 & 46411 & 46406 & 0.999892266 & 30941 & 30936 & 0.9998384 \\
\hline 6 & 150837 & 150831 & 0.999960222 & 402234 & 402228 & 0.9999850 \\
\hline 7 & 1960891 & 1960884 & 0.99999643 & 522943 & 5229036 & 0.999998661 \\
\hline
\end{tabular}

Here again, the thing to be observed is the number of messages with the same number of parity checks, correcting the limited patterns.

Example 3. Next let us consider the S-K partition of $Z_{q}$ given by

$$
P_{2}=\left\{B_{0}, B_{1}, B_{2}, B_{3}\right\},
$$

where

$$
B_{0}=\{0\}, B_{1}=\{1,2,11,12\} \text { and } B_{2}=\{3,4,9,10\}, B_{3}=\{5,6,7,8\} \text {, }
$$

now for an $(n, k)$ code correcting single error of weight 1 , then (by Theorem 1$)$ we have

$$
1+\left(\begin{array}{l}
n \\
1
\end{array}\right)\left|B_{1}\right| \leq q^{n-k} \text {, where } n-k=r \text { or } n \leq \frac{q^{r}-1}{\left|B_{1}\right|},
$$

and the numerical values are as given in Table 3.

Table 3

\begin{tabular}{|l|l|l|l|l|l|l|}
\hline \multirow{2}{*}{$r$} & \multicolumn{3}{|c|}{$\begin{array}{c}\text { Upper bound on } n \\
\text { with S-K single error }\end{array}$} & \multicolumn{3}{c|}{$\begin{array}{c}\text { Upper bound on } n \\
\text { with a Hamming-single error }\end{array}$} \\
\cline { 2 - 7 } & $n$ & $k$ & $R$ & $n$ & $k$ & $R$ \\
\hline 1 & 3 & 2 & 0.666666 & 1 & 0 & 0 \\
\hline 2 & 42 & 40 & 0.952380952 & 14 & 12 & 0.85714 \\
\hline 3 & 549 & 546 & 0.994535519 & 183 & 180 & 0.983606 \\
\hline 4 & 7140 & 7136 & 0.999439775 & 2830 & 2826 & 0.998586 \\
\hline 5 & 92823 & 92818 & 0.999946134 & 30941 & 30936 & 0.9998384 \\
\hline 6 & 1206702 & 1206696 & 0.999995027 & 402234 & 402228 & 0.9999850 \\
\hline 7 & 15687129 & 15687122 & 0.999999553 & 522943 & 5229036 & 0.999998661 \\
\hline
\end{tabular}

Suppose that we want to correct an error in 1 position of weight 1 and weight 2, then (by Theorem 2) we have 


$$
1+\left(\begin{array}{l}
n \\
1
\end{array}\right)\left(\left|B_{1}\right|+\left|B_{2}\right|\right) \leq q^{n-k} \text { or } n \leq \frac{q^{r}-1}{\left(\left|B_{1}\right|+\left|B_{2}\right|\right)},
$$

for different values of $r$, the upper-bound on $n$ for $(n, k), r=n-k$, is given in Table 4.

Table 4
\begin{tabular}{|l|l|l|l|l|l|l|}
\hline \multirow{3}{*}{$r$} & \multicolumn{3}{|c|}{$\begin{array}{c}\text { Upper bound on } n \\
\text { with S-K single error }\end{array}$} & \multicolumn{3}{c|}{$\begin{array}{c}\text { Upper bound on } n \\
\text { with a Hamming-single error }\end{array}$} \\
\cline { 2 - 7 } & $n$ & $k$ & $R$ & $n$ & $k$ & $R$ \\
\hline 1 & 1 & 0 & 0 & 1 & 0 & 0 \\
\hline 2 & 21 & 19 & 0.90476 & 14 & 12 & 0.85714 \\
\hline 3 & 274 & 271 & 0.989051094 & 183 & 180 & 0.983606 \\
\hline 4 & 3570 & 3566 & 0.99887955 & 2830 & 2826 & 0.998586 \\
\hline 5 & 46411 & 46406 & 0.99989227 & 30941 & 30936 & 0.9998384 \\
\hline 6 & 150837 & 150831 & 0.999960222 & 402234 & 402228 & 0.9999850 \\
\hline 7 & 1960891 & 1960884 & 0.99999643 & 522943 & 5229036 & 0.999998661 \\
\hline
\end{tabular}

Example 4. Here we shall illustrate some numerical bounds on $n$ when instead of a single error, the errors in any two or less positions are considered of weight 1 and then again either of weight 1 or 2 .

Let $Z_{q}=\{0,1,2,3,4,5,6,7,8,9,10,11,12\}, q=13$, consider S-K partition of $Z_{q}$, given by

$$
P=\left\{B_{0}, B_{1}, B_{2}, B_{3}\right\}
$$

where

$$
B_{0}=\{0\}, B_{1}=\{1,2,11,12\}, B_{2}=\{3,4,9,10\} \text { and } B_{3}=\{5,6,7,8\} .
$$

Case A. Suppose that we want to correct the error in two positions of weight 1 , then (by Theorem 1) we have

$$
\begin{gathered}
1+\left(\begin{array}{l}
n \\
1
\end{array}\right)\left|B_{1}\right|+\left(\begin{array}{l}
n \\
2
\end{array}\right)\left|B_{1}\right|^{2} \leq q^{n-k}, \\
n\left|B_{1}\right|+\frac{n(n-1)}{2}\left|B_{1}\right|^{2} \leq q^{r}-1, \\
4 n+8 n(n-1) \leq q^{r}-1, \\
4 n(2 n-1) \leq q^{r}-1 \text { or } n(2 n-1) \leq \frac{q^{r}-1}{4} .
\end{gathered}
$$

This is a quadratic Diophantine in-equation. By trial it is quite possible to find the largest value of $n$ satisfying this inequality. For different values of $r$, the upperbound on $n$ for $(n, k), r=n-k$, is given in Table 5 . 
Table 5

\begin{tabular}{|c|c|c|c|c|c|c|}
\hline \multirow[t]{2}{*}{$r$} & \multicolumn{3}{|c|}{$\begin{array}{c}\text { Upper bound on } n \\
\text { with S-K single error } \\
\left(n(2 n-1) \leq \frac{q^{r}-1}{4}\right)\end{array}$} & \multicolumn{3}{|c|}{$\begin{array}{c}\text { Upper bound on } n \\
\text { with a Hamming-single } \\
\text { error } \\
\left(n(6 n-5) \leq \frac{q^{r}-1}{12}\right)\end{array}$} \\
\hline & $n$ & $k$ & $R$ & $n$ & $k$ & $R$ \\
\hline 1 & 1 & 0 & 0 & 1 & 0 & 0 \\
\hline 2 & 4 & 2 & 0.5 & 2 & 0 & 0 \\
\hline 3 & 16 & 13 & 0.8125 & 5 & 2 & 0.4 \\
\hline 4 & 60 & 56 & 0.93333 & 20 & 16 & 0.8 \\
\hline 5 & 215 & 210 & 0.976744186 & 72 & 67 & 0.93056 \\
\hline 6 & 777 & 771 & 0.992277992 & 259 & 253 & 0.97683 \\
\hline 7 & 2800 & 2793 & 0.9975 & 933 & 926 & 0.9925 \\
\hline
\end{tabular}

Case B. Next, let us consider the errors in two positions of weight 1, then (by Theorem 1) we have

$$
\begin{gathered}
1+\left(\begin{array}{l}
n \\
1
\end{array}\right)\left(\left|B_{1}\right|+\left|B_{2}\right|\right)+\left(\begin{array}{l}
n \\
2
\end{array}\right)\left(\left|B_{1}\right|+\left|B_{2}\right|\right)^{2} \leq q^{n-k}, \\
1+8 n+32 n(n-1) \leq q^{n-k}, \\
8 n+32 n(n-1) \leq q^{n-k}-1 \text { or } 8 n(4 n-3) \leq q^{n-k}+1, \\
n(4 n-3) \leq \frac{q^{n-k}+1}{8},
\end{gathered}
$$

for different values of $r$, the upper-bound on $n$ for $(n, k), r=n-k$, is given in Table 6.

Table 6

\begin{tabular}{|l|l|l|l|l|l|l|}
\hline & \multicolumn{2}{|c|}{$\begin{array}{c}\text { Upper bound on } n \\
\text { with S-K single error }\end{array}$} & \multicolumn{2}{c|}{$\begin{array}{c}\text { Upper bound on } n \\
\text { with a Hamming-single } \\
\text { error }\end{array}$} \\
& \multicolumn{3}{|c|}{$\left(n(2 n-1) \leq \frac{q^{r}-1}{4}\right)$} & \multicolumn{3}{r|}{$\left(n(6 n-5) \leq \frac{q^{r}-1}{12}\right)$} \\
\cline { 2 - 7 } & $n$ & $k$ & $R$ & $n$ & $k$ & $R$ \\
\hline 1 & 1 & 0 & 0 & 1 & 0 & 0 \\
\hline 2 & 2 & 0 & 0 & 2 & 0 & 0 \\
\hline 3 & 8 & 5 & 0.625 & 5 & 2 & 0.4 \\
\hline 4 & 30 & 26 & 0.86666 & 20 & 16 & 0.8 \\
\hline 5 & 108 & 103 & 0.953704 & 72 & 67 & 0.93056 \\
\hline 6 & 388 & 382 & 0.984536 & 259 & 253 & 0.97683 \\
\hline 7 & 443 & 436 & 0.984199 & 933 & 926 & 0.9925 \\
\hline
\end{tabular}




\section{Concluding remarks}

In this paper we have introduced the idea of limited patterns of errors and have obtained results on the upper bounds on word lengths for specified limited patterns of errors. The use of S-K partitions and distances provides a choice of metrics matching the channel characteristics.

It seems quite possible to extend this study to other bounds and to constructions of codes correcting limited patterns of errors.

\section{References}

1. B e r l e k a m p, E. R. Algebraic Coding Theory. New York, McGraw-Hill, 1968.

2. K a u s h i k, M. L. A New Metric in the Study of Error Correcting Codes. Ph. D. Thesis, University of Delhi, 1979.

3. P e t e r s o n, W. W., E. J. W e l d o n, Jr. Error-Correcting Codes. 2nd Ed. Cambridge, Mass., MIT Press, 1972.

4. S h a r ma, B. D., M. L. Ka u sh i k. Error Correcting Codes Through a New Metric. - In: 41st Annual Conf. Intern. Stat. Inst., New Delhi, 1977.

5. S h a r m a, B. D., M. L. K a u s h i k. Algebra of Sharma and Kaushik's Metric Inducing Partitions of Zq. - J. Combin. Information System Science, Vol. 11, 1986, 19-32.

6. S h a rm a, B. D., G. D i a l. Some Tighter Bounds on Code Size with Sharma-Kaushik Metrics. In: Intern. Conf. on Math., Mao, Menorca, June, 1987, 15-17. 